\title{
Kidney and lung injury in rats following acute diquat exposure
}

\author{
YUZHENG WU $^{1,2}$, SIQI CUI ${ }^{1,2}$, WENJUN WANG ${ }^{1}$, TIANZI JIAN ${ }^{1}$, BAOTIAN KAN ${ }^{3}$ and XIANGDONG JIAN ${ }^{1,2}$ \\ ${ }^{1}$ Department of Poisoning and Occupational Diseases, Emergency Department, \\ Qilu Hospital of Shandong University; ${ }^{2}$ School of Public Health, Shandong University; \\ ${ }^{3}$ Department of Geriatric Medicine, Qilu Hospital of Shandong University, Jinan, Shandong 250012, P.R. China
}

Received October 26, 2021; Accepted January 13, 2022

DOI: $10.3892 / \mathrm{etm} .2022 .11201$

\begin{abstract}
Diquat (1,1'-ethylene-2,2'-bipyridylium) is a type of widely used agricultural chemical, whose toxicity results in damage to numerous tissues, including the lung, liver, kidney and brain. The aim of the present study was to establish a rat model of acute diquat exposure and explore the relationship between diquat concentration, and kidney and lung injury, in order to provide an experimental basis for clinical treatment. A total of 140 healthy adult male Wistar rats were randomly divided into control and exposure groups. The diquat solution was administered intragastrically to the exposure group at $1 / 2$ of the lethal dose $(140 \mathrm{mg} / \mathrm{kg})$. An equal volume of water was administered to the control group. The dynamic changes in the plasma and tissue diquat levels were quantitatively determined at $0.5,1,2,4,8,16$ and $24 \mathrm{~h}$ following exposure using liquid chromatography mass spectrometry. The content of hydroxyproline (HYP) in the lung tissues, as well as the levels of blood urea nitrogen $(\mathrm{BUN})$, creatinine $(\mathrm{Cr})$, uric acid (UA), kidney injury molecule-1 (KIM-1) and tumor growth factor (TGF)- $\beta 1$, were detected using western blot analysis at every time point. Lung and kidney morphology were also assessed. Electron microscopy showed that the degree of renal damage gradually increased with time. Vacuolation gradually increased, some mitochondrial bilayer membrane structures disappeared and lysosomes increased. The lung tissue damage was mild, and the cell membrane integrity and organelles were damaged to varying degrees. The plasma and organ levels of diquat peaked at $\sim 2 \mathrm{~h}$, followed by a steady decrease, depending on the excretion rate. Over time, the serum concentrations of UA, BUN, Cr and KIM-1 were all significantly increased $(\mathrm{P}<0.05)$. Serum KIM-1 in rats was increased after $0.5 \mathrm{~h}$, and was significantly increased after $4 \mathrm{~h}$, suggesting that KIM-1 is an effective predictor of early renal injury. Early TGF- $\beta 1$ expression was clearly observed in renal tissue, while no clear
\end{abstract}

Correspondence to: Dr Xiangdong Jian, Department of Poisoning and Occupational Diseases, Emergency Department, Qilu Hospital of Shandong University, 107 Wenhuaxi Road, Jinan, Shandong 250012, P.R. China

E-mail: jianxiangdongvip@vip.163.com

Key words: animal model, diquat, acute toxicity, kidney injury
TGF- $\beta 1$ expression was observed in the lung tissue. In conclusion, the concentration of diquat in the serum and tissue of rats with acute diquat poisoning peaked at an early stage and then rapidly decreased. The renal function damage and pathological changes persisted, the lung tissue was slightly damaged with inflammatory cell infiltration, and early pulmonary fibrosis injury was not obvious.

\section{Introduction}

Diquat (1,1'-ethylene-2,2'-bipyridylium) is a type of widely used agricultural chemical, whose toxicities result in the damage to numerous tissues, including the lung, liver, kidney and brain (1). Diquat is a herbicide that started to be widely used in agricultural production following the ban of paraquat (2). As a result, the incidence of rapid acute diquat poisoning has increased in recent years. Diquat is very toxic to humans, as it leads to damage of the function of multiple organs, and can even prove life-threatening $(3,4)$. Diquat is distributed through the bloodstream to the organs and tissues of the entire body. The liver and kidney contain the highest concentrations of diquat (5). Research has also found that bile contains diquat, which indicates that bile may be an effective way of lowering the concentration of diquat in the serum and tissues of the human body long after ingestion (6). Redox cycling and consequent reactive oxygen species (ROS) generation are believed to be key cytotoxic mechanisms induced by these bipyridyl herbicides. The diquat radicals are also capable of reacting with a variety of nucleophiles in cells, causing direct damage to cellular components $(2,7)$. To date, the treatment method for diquat poisoning and satisfactory therapeutic effects remain in the exploratory stages. There is no specific antidote for rapid poisoning with diquat, as well as no clear therapeutic means of treatment (4). Key factors that determine the prognosis of diquat poisoning include whether the patient goes to the hospital after poisoning, the timely and effective gastrointestinal treatment and application of blood purification technology, as well as the timely application of glucocorticoids to protect organ function $(3,8)$. Although human exposure to diquat can cause dysfunction or even multiple organ failure, the liver and kidneys are the major organs involved, mainly due to the high accumulation of diquat in those organs after poisoning (9). It is worth noting that diquat is mainly metabolized in the kidneys; thus, the kidneys are the main target organ after poisoning $(9,10)$. In 
the present study, a rat model of acute diquat exposure was established following gavage administration to further unveil the distribution characteristics, metabolism and organ damage of diquat in the body.

\section{Materials and methods}

Experimental animals, dosing, and blood and tissue collection. Healthy adult SPF male Wistar rats aged 6-8 weeks and weighing 240-260 g were provided by the Experimental Animal Center of Shandong University (Jinan, China) [certificate no. SCXK (Lu) 20130009]. Ethical approval for this experiment was obtained from the Ethics Committee of Preventive Medicine of Shandong University (no. 20180225/February 12, 2018). The rats were allowed to eat and drink freely, and the environmental conditions of the room were set to maintain a relative humidity of $40-70 \%$, a temperature of $\sim 26^{\circ} \mathrm{C}$ and a 12-h light/dark cycle. The rats were fasted overnight $(\sim 16 \mathrm{~h})$, and were allowed free access to water prior to treatment.

A total of 140 healthy adult male Wistar rats were randomly divided into the control and exposure groups. The dose of the diquat solution intragastrically administered to the exposure groups was $140 \mathrm{mg} / \mathrm{kg}$ (50\% of the median lethal dose) (11). An equal volume of normal saline solution was administered to the control group $(n=70)$. The dynamic changes in the plasma and tissue diquat levels were quantitatively determined by liquid chromatography mass spectrometry at 0.5 , $1,2,4,8,16$ and $24 \mathrm{~h}$ after poisoning ( $\mathrm{n}=10$ per time point). Approximately $5 \mathrm{ml}$ of blood was taken from the inferior vena cava for serological tests. Blood was collected following cervical dislocation under anesthesia. The blood was placed in a short purple tube (containing an EDTA anticoagulant). Following centrifugation, the plasma was collected and frozen at $-80^{\circ} \mathrm{C}$ for serum concentration and renal function analyses. Left lung and left kidney tissues were collected to determine the tissue diquat content using electron microscopy (Zeiss Axio Scope A1 Microscope; Carl Zeiss AG) and for western blot analysis. The right lung and right kidney tissues were used for pathological examination and fixed in a paraformaldehyde solution with a mass volume fraction of $4 \%$. Conventional paraffin was used for embedding, and the tissue was sectioned. Following hematoxylin and eosin (H\&E) and Masson staining, the sections were observed using the IX53 and DP73 upright fluorescence microscopes (Olympus Corp.). In addition, the lung tissue was used to determine the hydroxyproline (HYP) content using a commercial kit from the Nanjing Jiancheng Bioengineering Institute (cat. no. A030-2).

Humane endpoints. The rats were anesthetized at the established time points $(0.5,1,2,4,8,16$ and $24 \mathrm{~h})$. The animals were anaesthetized through an intraperitoneal injection of $10 \%$ chloral hydrate $(300 \mathrm{mg} / \mathrm{kg})$. The rats were euthanized by cervical dislocation immediately after anesthesia. At that time, peritonitis had not developed in the rats.

Establishment of the standard curve. The peak area of diquat was used as the $y$-coordinate, the concentration of diquat was plotted on the abscissa and the weighting coefficient $\left(1 / \mathrm{x}^{2}\right)$ was used to obtain a standard curve.
Detection of various kidney indexes. Creatinine $(\mathrm{Cr})$, blood urea nitrogen (BUN) and uric acid (UA) were detected using an automatic biochemical analyzer [Cobas ${ }^{\circledR} 8000$ detection system (775 module); Roche Diagnostics GmbH] at Qilu Hospital, Shandong University (Jinan, China). BUN was used for the urease method, and UA and $\mathrm{Cr}$ were used for the enzyme method.

ELISA and western blot analysis. The tissue block was washed with pre-cooled PBS for 2-3 times, two 3-mm homogenization beads were added, 10 times the tissue volume of lysis buffer (Servicebio G2002) was added for homogenization, and then the lysis buffer was placed on ice for $30 \mathrm{~min}$. After complete lysis, centrifugation at $12,000 \mathrm{rpm}$ was carried out for $10 \mathrm{~min}$ at $4^{\circ} \mathrm{C}$, the supernatant was collected, and the protein concentration was measured with a BCA protein concentration test kit. Reduced protein loading buffer was added to the protein solution at a ratio of $4: 1$, denatured in a boiling water bath for $15 \mathrm{~min}$, and stored at $-20^{\circ} \mathrm{C}$ for later use. The proteins were separated by $10 \%$ SDS-PAGE, transferred to PVDF membranes, blocked with $5 \%$ skim milk in TBST buffer (20\% Tween) for $30 \mathrm{~min}$ at room temperature, and then incubated with the primary antibody overnight at $4^{\circ} \mathrm{C}$. The primary antibody dilution ratio was $1: 1,000$ and it included KIM-1 (Abbexa, cat. no. ABX102459), TGF- $\beta 1$ (Abcam, cat. no. AB92486), and actin (Servicebio). Membranes were then washed three times with TBST and incubated with secondary antibodies peroxidase-conjugated goat anti-rabbit IgG (H+L) (Servicebio, cat. no. GB23303), peroxidase-conjugated donkey anti-goat IgG (H+L) (Servicebio, cat. no. GB23404), peroxidase-conjugated goat anti-mouse $\operatorname{IgG}(\mathrm{H}+\mathrm{L})$ (Servicebio, cat. no. GB23301), peroxidase-conjugated goat anti-rat $\mathrm{IgG}(\mathrm{H}+\mathrm{L})$ (Servicebio, cat. no. GB23302) at room temperature. The protein bands were observed using ECL (Servicebio, cat. no. G2014). The semi-quantitative expression was measured by densitometry analysis using AlphaEaseFC 5.0 software (Alpha Innotech), and normalized to the expression of actin.

Statistical analyses. All experiments were performed independently at least 3 times. Data are presented as mean \pm standard deviation of three replicates. SPSS17.0 statistical analysis software (SPSS Inc.) was used to conduct normality test, variance homogeneity test, one-way ANOVA and repeated measures analysis of variance for the experimental data. For pairwise comparisons, Dunnett's T3 was used when the variances were unequal, and LSD was used when the variances were homogeneous.

\section{Results}

$H \& E$ staining in the lung and renal tissues. Fig. 1 shows $\mathrm{H} \& \mathrm{E}$ staining in lung tissues in the control (a), and at times 0.5 (b), 1 (c), 2 (d), 4 (e), 8 (f), 16 (g) and $24 \mathrm{~h}$ (h) after diquat exposure. In the exposure groups, H\&E staining showed that the alveolar walls were dilated and hyperaemic, and the alveolar spaces were slightly widened. Infiltration by polymorphonuclear leukocytes and mononuclear macrophages was visible, and the alveolar cavities were clean, cell-free and structurally intact. Inflammatory cell infiltration between the pulmonary alveoli was comparable 8,16 and $24 \mathrm{~h}$ after administration, and the pulmonary alveoli were wider than previously measured. Part of the alveolar structure was disordered, and the alveolar cavities had disappeared (Fig. 1). 

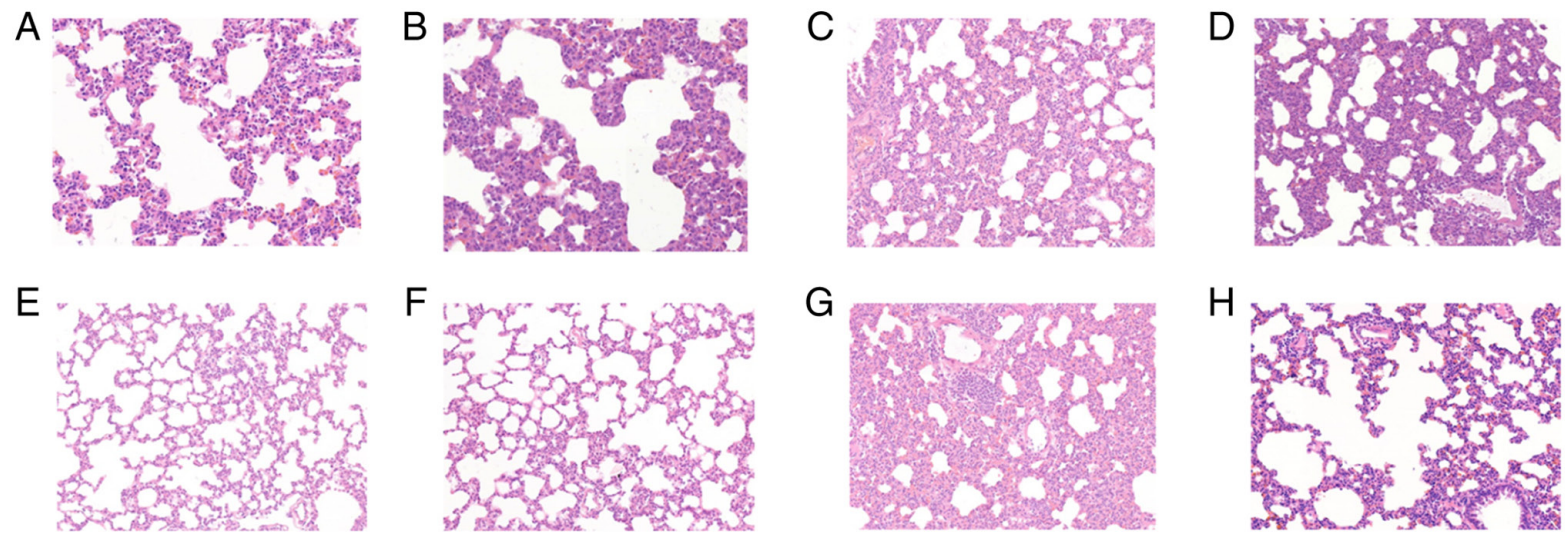

Figure 1. Results of hematoxylin and eosin (H\&E) staining in lung tissues. Light microscopic observation of lung pathological sections at each time point (A-H shows the control, and at times $0.5,1,2,4,8,16$ and $24 \mathrm{~h}$ in lung tissue after diquat exposure; magnification, x200). (A) In the control group, the alveolar walls were dilated and hyperaemic, and the alveolar spaces were slightly widened. Infiltration by polymorphonuclear leukocytes and mononuclear macrophages was visible. At (B) 0.5 , (C) 1, (D) 2 and (E) $4 \mathrm{~h}$, the alveolar cavities were clean, cell-free and structurally intact. At (F) 8 , (G) 16 and (H) $24 \mathrm{~h}$, the pulmonary alveoli were wider than previously measured. Part of the alveolar structure was disordered, and the alveolar cavities had disappeared.
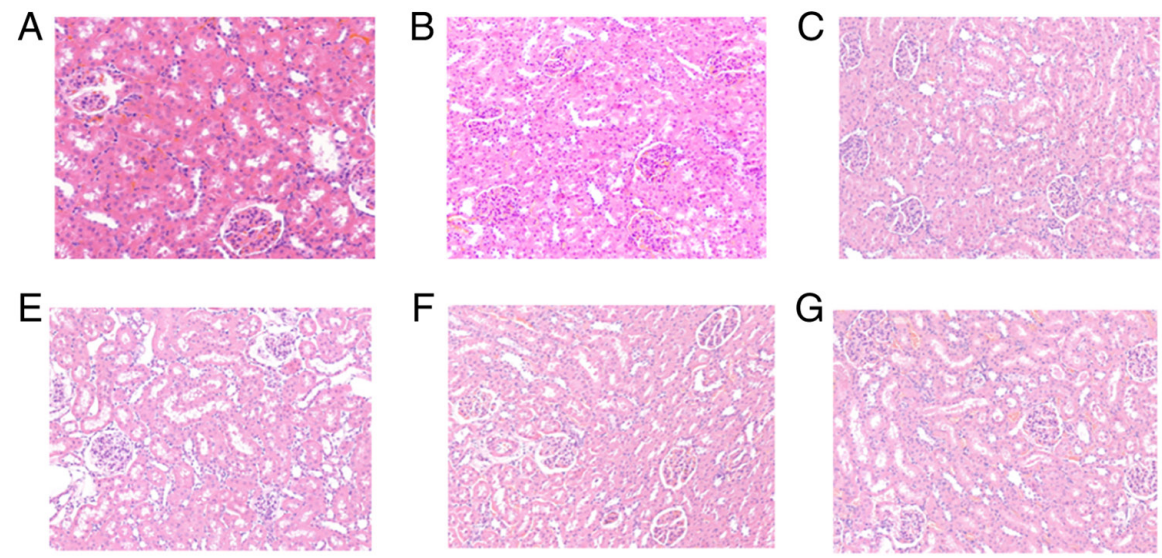

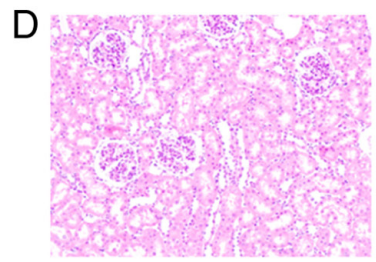

$\mathrm{H}$

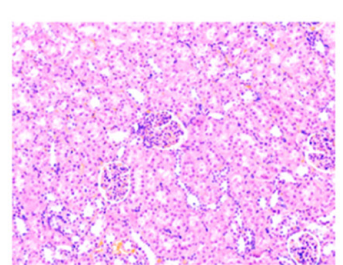

Figure 2. Results of hematoxylin and eosin (H\&E) staining in renal tissues. Light microscopic observation of renal pathological sections at each time point (A-H shows the control, and at times $0.5,1,2,4,8,16$ and $24 \mathrm{~h}$ in renal tissue after diquat exposure; magnification, $\mathrm{x} 200$ ). (A) In the control group, there was no congestion observed at any time point. The glomerular basement membrane was intact, and the renal tubules had clear borders, with no exfoliated cells in the lumen. Pathological changes, such as inflammatory cell infiltration and fibrous tissue hyperplasia, were rare in the stroma. At (B) 0.5 , (C) 1 and (D) $2 \mathrm{~h}$, renal tubular epithelial cells exhibited hydropic degeneration, and a small number of casts were observed in the lumen. At (E) 4 and (F) 8 h, the glomerular mesentery basement membrane was slightly thickened and partial glomerular atrophy was observed. The renal tissue exhibited oedema, and the kidney tubules were notably widened. At (G) 16 and $(\mathrm{H}) 24 \mathrm{~h}$, mesenchymal inflammatory cell infiltration was observed.

Fig. 2 shows the results of H\&E staining in renal tissues. Light microscopic observation of renal pathological sections in the control (a), and at times 0.5 (b), 1 (c), 2 (d), 4 (e), 8 (f), $16(\mathrm{~g})$ and $24 \mathrm{~h}(\mathrm{~h})$ after diquat exposure is shown. Under microscopic examination, the renal interstitium was not found to be congested in the control group at any time point. The glomerular basement membrane was intact, and the renal tubules had clear borders, with no exfoliated cells in the lumen. Pathological changes, such as inflammatory cell infiltration and fibrous tissue hyperplasia, were rare in the stroma. In the exposure group, renal tubular epithelial cells exhibited hydropic degeneration, and a small number of casts were observed in the lumen. The glomerular mesentery basement membrane was slightly thickened and partial glomerular atrophy was observed. The renal tissue exhibited oedema, and the kidney tubules were notably widened. After 16 and $24 \mathrm{~h}$, mesenchymal inflammatory cell infiltration was observed (Fig. 2).
Electron microscopy observation of the lung tissue. After $0.5 \mathrm{~h}$, a large number of organelles were observed in the cytoplasm of the lung type II alveolar cells using electron microscopy (Fig. 3), and a number of organelles were shown to be swollen. After $2 \mathrm{~h}$, a small number of microvilli appeared locally in the cell membrane and an abundance of organelles were observed in the cytoplasm. The abundance of organelles were notably swollen and dilated after $8 \mathrm{~h}$. After $16 \mathrm{~h}$, the cell membrane of the type II lung alveolar cells had partially disappeared. After $24 \mathrm{~h}$, the cell membrane was partially blurred, and abundant and slightly swollen organelles (slightly milder than at $16 \mathrm{~h}$ ) were observed in the cytoplasm.

Electron microscopy observation of renal tissues. Electron microscopy of renal tissues (Fig. 4) revealed relatively intact renal tubular epithelial cell membranes after $2 \mathrm{~h}$, with an abundance of organelles and numerous vacuoles in the cytoplasm. After $4 \mathrm{~h}$, the renal tubular epithelial cells were relatively 

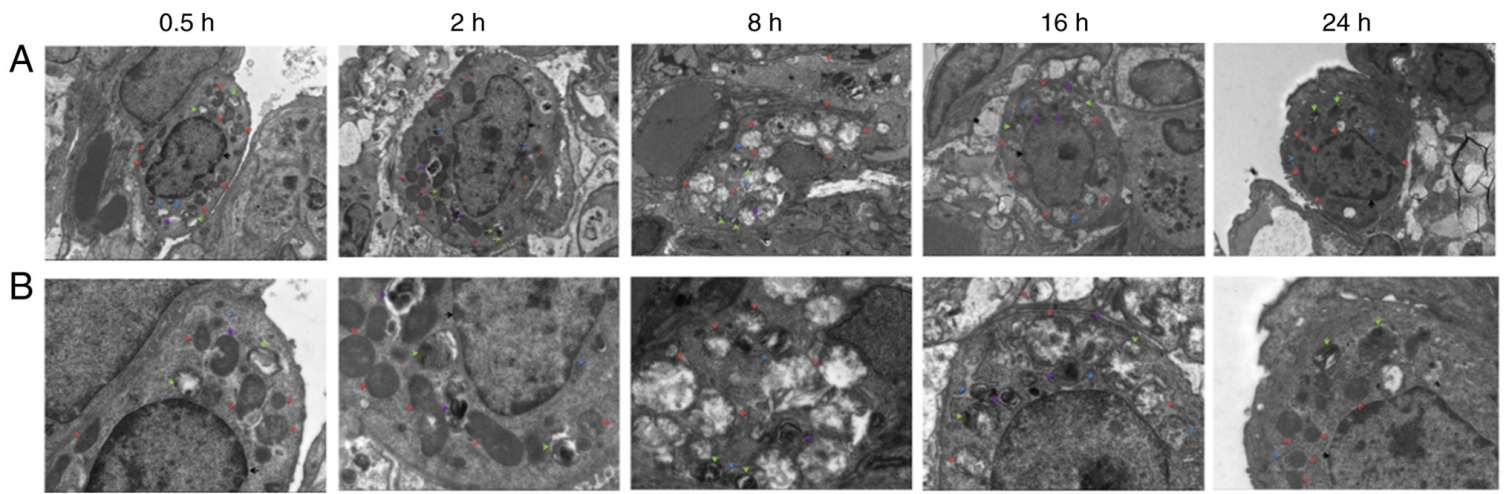

Figure 3. Electron microscopy observation of lung tissue at each time point. (A) Electron microscopy of lung tissue at $0.5,2,8,16$ and $24 \mathrm{~h}$ after diquat exposure (magnification, x3,000; scale bar, $5 \mu \mathrm{m}$ ). (B) Electron microscopy of lung tissue at 0.5, 2, 8, 16 and $24 \mathrm{~h}$ after diquat exposure (magnification, x7,000; scale bar, $2 \mu \mathrm{m})$.
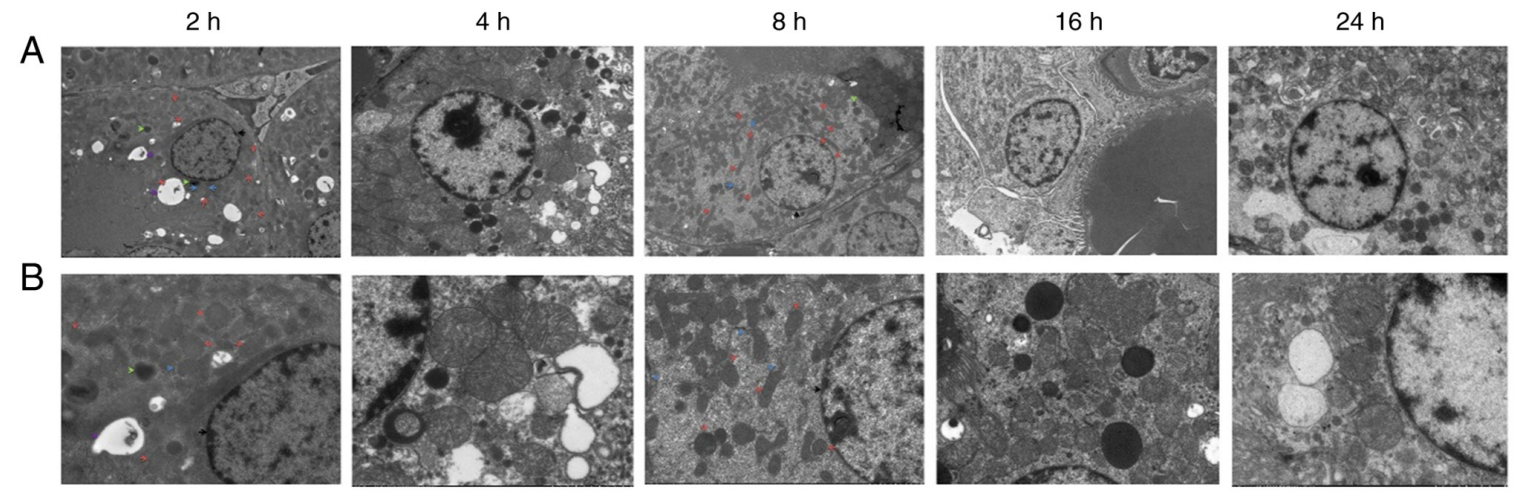

Figure 4. Electron microscopy observation of renal tissue at each time point. (A) Electron microscopy of renal tissue at 2, 4, 8, 16 and $24 \mathrm{~h}$ after diquat exposure (magnification, x2,000; scale bar $5 \mu \mathrm{m}$ ). (B) Electron microscopy of renal tissue at 2, 4, 8, 16 and $24 \mathrm{~h}$ after diquat exposure (magnification, x5,000; scale bar, $2 \mu \mathrm{m})$.
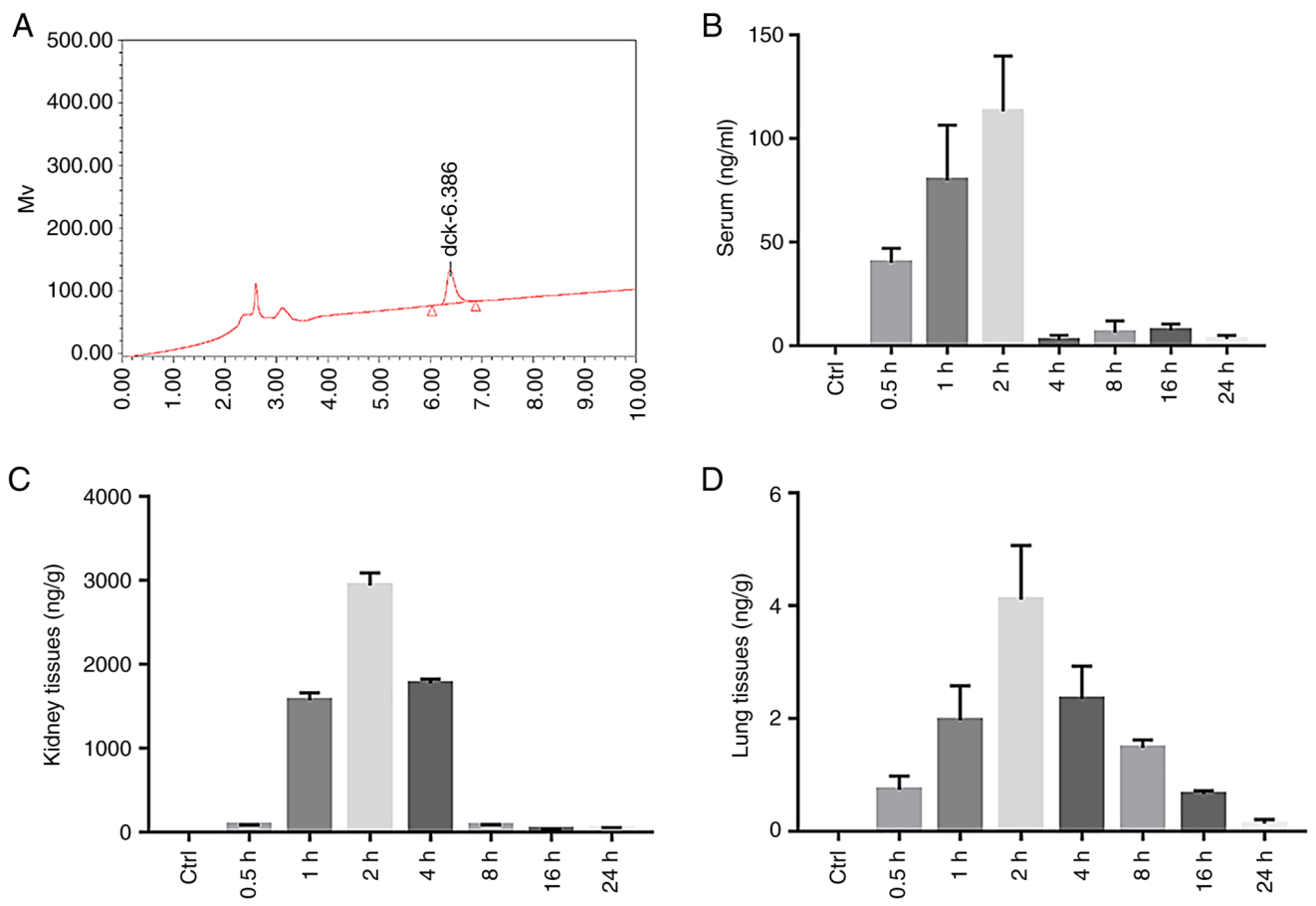

Figure 5. The content of diquat in the plasma, kidney and lung tissues of rats. (A) HPLC of the diquat standard. (B) Histogram of the serum diquat concentration in the Control (Ctrl) and Experimental group (at 0.5, 1, 2, 4, 8, 16 and $24 \mathrm{~h}$ after diquat exposure). (C) Histogram of kidney diquat concentration in the Control (Ctrl) and Experimental group (at 0.5, 1, 2, 4, 8, 16 and $24 \mathrm{~h}$ after diquat exposure). (D) Histogram of lung diquat concentration in the Control (Ctrl) and Experimental group (at 0.5, 1, 2, 4, 8, 16 and $24 \mathrm{~h}$ after diquat exposure). 
Table I. Regression equations of plasma and tissues.

\begin{tabular}{llr}
\hline Biological examination & Regression equation & Correlation coefficient $r$ \\
\hline Plasma & $\mathrm{y}=332.855 \mathrm{x}+1,395.76$ & 0.9941 \\
Renal & $\mathrm{y}=9,450 \mathrm{x}+1,650$ & 0.9993 \\
Lung & $\mathrm{y}=24,440 \mathrm{x}+1,050$ & 0.9968 \\
\hline
\end{tabular}

Table II. Concentration of diquat at every time point after infection $(\mathrm{n}=10, \pm \mathrm{SD}, \mathrm{ng} / \mathrm{ml})$.

\begin{tabular}{|c|c|c|c|c|c|c|c|}
\hline $\begin{array}{l}\text { Group } \\
\text { Control group }\end{array}$ & $\begin{array}{l}0.5 \mathrm{~h} \\
\mathrm{BLQ}\end{array}$ & $\begin{array}{c}1 \mathrm{~h} \\
\mathrm{BLQ}\end{array}$ & $\begin{array}{c}2 \mathrm{~h} \\
\mathrm{BLQ}\end{array}$ & $\begin{array}{c}4 \mathrm{~h} \\
\mathrm{BLQ}\end{array}$ & $\begin{array}{c}8 \mathrm{~h} \\
\mathrm{BLQ}\end{array}$ & $\begin{array}{l}16 \mathrm{~h} \\
\mathrm{BLQ}\end{array}$ & $\begin{array}{l}24 \mathrm{~h} \\
\mathrm{BLQ}\end{array}$ \\
\hline \multicolumn{8}{|l|}{ Exposure group } \\
\hline Serum & $40.14 \pm 6.86^{\mathrm{a}}$ & $79.91 \pm 26.71^{\mathrm{a}}$ & $113.20 \pm 26.72^{\mathrm{a}}$ & $2.87 \pm 2.17$ & $6.41 \pm 5.62$ & $7.49 \pm 3.08$ & $3.26 \pm 1.85$ \\
\hline Kidney tissues & $84.17 \pm 3.39$ & $1,575.70 \pm 86.68^{a}$ & $2,940.38 \pm 151.49^{a}$ & $1,775.69 \pm 49.73^{\mathrm{a}}$ & $77.96 \pm 13.48$ & $34.38 \pm 4.35$ & $52.11 \pm 2.97$ \\
\hline Lung tissues & $0.74 \pm 0.24$ & $1.97 \pm 0.61^{\mathrm{a}}$ & $4.11 \pm 0.96^{\mathrm{a}}$ & $2.35 \pm 0.58^{\mathrm{a}}$ & $1.48 \pm 0.14$ & $0.66 \pm 0.06$ & $0.14 \pm 0.07$ \\
\hline
\end{tabular}

${ }^{\text {a }}<0.01$, compared with the control group. BLQ, below the detection lower limit.

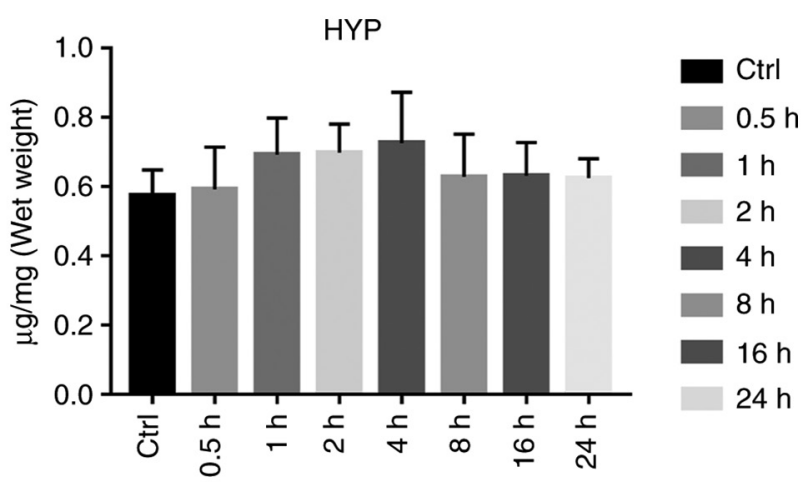

Figure 6. Histogram of lung hydroxyproline (HYP) levels in rats in the Control (Ctrl) and Experimental group (at 0.5, 1, 2, 4, 8, 16 and $24 \mathrm{~h}$ after diquat exposure).

intact, and an abundance of organelles was observed in the cytoplasm. The nuclear membrane was intact, the nucleolus was clearly visible, and the level of heterochromatin had increased. Mitochondrial swelling and cytoplasmic vacuolization were observed. After $8 \mathrm{~h}$, the amount of heterochromatin and lysosomes increased. After $16 \mathrm{~h}$, the mitochondria were swollen, part of the mitochondrial cristae was fractured, the podocyte feet were protruded and fused, and the basement membrane exhibited dense deposition. After $24 \mathrm{~h}$, cavitation bubbles appeared; the bilayer membrane structure of some mitochondria had disappeared, podocytic processes of Sertoli cells were fused, a dense basement membrane deposition was observed, and the number of lysosomes had increased.

Diquat levels in the plasma, kidney and lung tissues of rats. The diquat peak was clear and specific under the chromatographic conditions used in this study, with a retention time of $6.386 \mathrm{~min}$ (Fig. 5A). The diquat peak area was considered as the $y$-coordinate, and the concentration of diquat as the $\mathrm{x}$-coordinate. The weighting factor $\left(1 / \mathrm{x}^{2}\right)$ was used to perform regression; the standard curve equation is listed in Table I. The standard curve of the diquat plasma sample exhibited a linear relationship at a concentration range of $0.100-5,000 \mathrm{ng} / \mathrm{ml}$, that of the renal sample at a concentration range of $0.1-200$, and that of the lung at a concentration range of 0.1-100. The lowest limit of detection, that is, the lowest point of the standard curve, was 0.1 . Results were compared with the standard curve. A content below the lower limit of detection (undetectable) was defined as below the limit of quantification.

The plasma concentrations of diquat significantly increased at $0.5,1$ and $2 \mathrm{~h}(\mathrm{P}<0.01)$ after administration, peaking at $2 \mathrm{~h}$, and then significantly decreasing by $4 \mathrm{~h}$, before slightly increasing again at 8 and $16 \mathrm{~h}$ (Fig. 5B and Table II). The concentration of diquat in the kidney was significantly increased at 1,2 and $4 \mathrm{~h}(\mathrm{P}<0.01)$ from administration (Table II), also peaking at $2 \mathrm{~h}$ (Fig. 5C and Table II). The concentration of diquat in the lung tissue was significantly increased at 1,2 and $4 \mathrm{~h}$ from administration (Fig. 5D and Table II), peaked at $2 \mathrm{~h}$ and then decreased.

HYP content determination in the lung tissues. HYP levels in the lung tissue reflects lung tissue collagen content, and is used as a parameter to assess the degree of pulmonary fibrosis. Compared with that in the control group, the HYP content in the lung tissues of the exposure group was not significantly increased, and variance analysis yielded no statistical significance $(\mathrm{P}>0.05$; Fig. 6 and Table III).

Renal function. The serum UA levels were significantly increased at $1,2,16$ and $24 \mathrm{~h}(\mathrm{P}<0.01)$, There was no apparent difference between the exposure and control groups at 0.5 , 4 and $8 \mathrm{~h}(\mathrm{P}>0.05)$. Following exposure, the serum BUN of each group was significantly increased, and the difference 


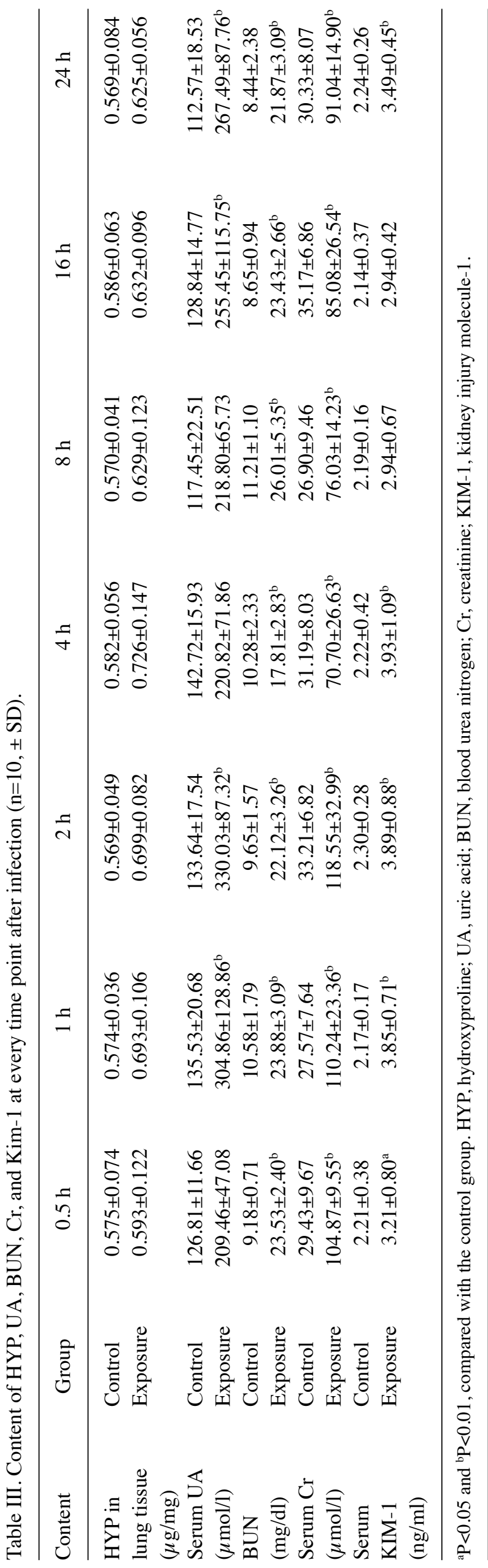

between the two groups was statistically significant $(\mathrm{P}<0.01)$. The serum $\mathrm{Cr}$ levels of the rats exposed to diquat were significantly increased from 0.5 to $24 \mathrm{~h}$, and the $\mathrm{Cr}$ content peaked at $2 \mathrm{~h}$. The $\mathrm{Cr}$ level of the exposure group was significantly higher from that of the control group $(\mathrm{P}<0.01$; Table III and Fig. 7A-C).

The serum KIM-1 levels were determined using ELISA and were found to be significantly increased at $0.5,1,2,4$ and $24 \mathrm{~h}$ from exposure $(\mathrm{P}<0.05)$. There was no significant difference between the groups after 8 and $16 \mathrm{~h}$ ( $\mathrm{P}>0.05$; Table III and Fig. 7D).

KIM-1 and TGF- $\beta 1$ expression in rat kidney and lung tissues were detected using western blot analysis. Compared with the control group, at $0.5,1,2,4,8,16$ and $24 \mathrm{~h}$ after exposure, the renal KIM- 1 and TGF- $\beta 1$ levels were increased, peaking at $2 \mathrm{~h}$ from exposure (Fig. 8A). The lung TGF- $\beta 1$ concentration was not significant (Fig. 8B).

\section{Discussion}

Diquat (1,1'-ethylene-2,2'-bipyridylium) is a type of widely used agricultural chemical, whose toxicities result in the damage to numerous tissues, including the lung, liver, kidney and brain (1). Diquat, a redox cycling compound, mediates its systemic toxicity through an enhanced production of free radicals. In vitro studies have shown that these compounds are potent generators of reactive oxygen species (ROS) by redox cycling and that they stimulate lipid peroxidation (5). However, in vivo research has failed to provide clear evidence of lipid peroxidation in response to exposure to these compounds (5). The role of ROS and lipid peroxidation in the toxic effects of diquat remains controversial. In the present study, a rat model of diquat poisoning was established using a one-time administration of diquat by gavage, and the dynamic changes in the levels of diquat in the blood, and various organs and tissues, were observed for $24 \mathrm{~h}$. The results showed that the plasma and organ levels of diquat peaked at $2 \mathrm{~h}$. They then decreased steadily according to different excretion rates. It is worth mentioning that the plasma diquat concentration increased again at $24 \mathrm{~h}$, which appeared to be linked to the reabsorption of renal tubules and collecting ducts, and enterohepatic circulation. Generally, the concentration of diquat was at its highest in the kidney, second highest in the plasma and lowest in the lung tissues, which is consistent with the metabolic effect of diquat, and the observed degree of injury to various organs in patients with diquat poisoning in the clinic.

The kidney is one of the main organs damaged by diquat poisoning. In the present study, rats with acute diquat exposure underwent significant renal function impairment in a short period of time, and the renal function indexes, specifically blood urea nitrogen (BUN) and creatinine $(\mathrm{Cr})$ were significantly higher in the exposure group than in the control group. Recent studies have shown that an increased uric acid (UA) is a strong independent biomarker for adverse outcomes in several diseases and conditions linked to increased oxidative stress and inflammation (12). The UA levels were not significantly increased in the 4-8 $\mathrm{h}$ group compared with the control group, which was considered to be linked to the transient decrease in UA levels through glomerular filtration, due to the strong compensatory function of the kidney during early renal injury. 

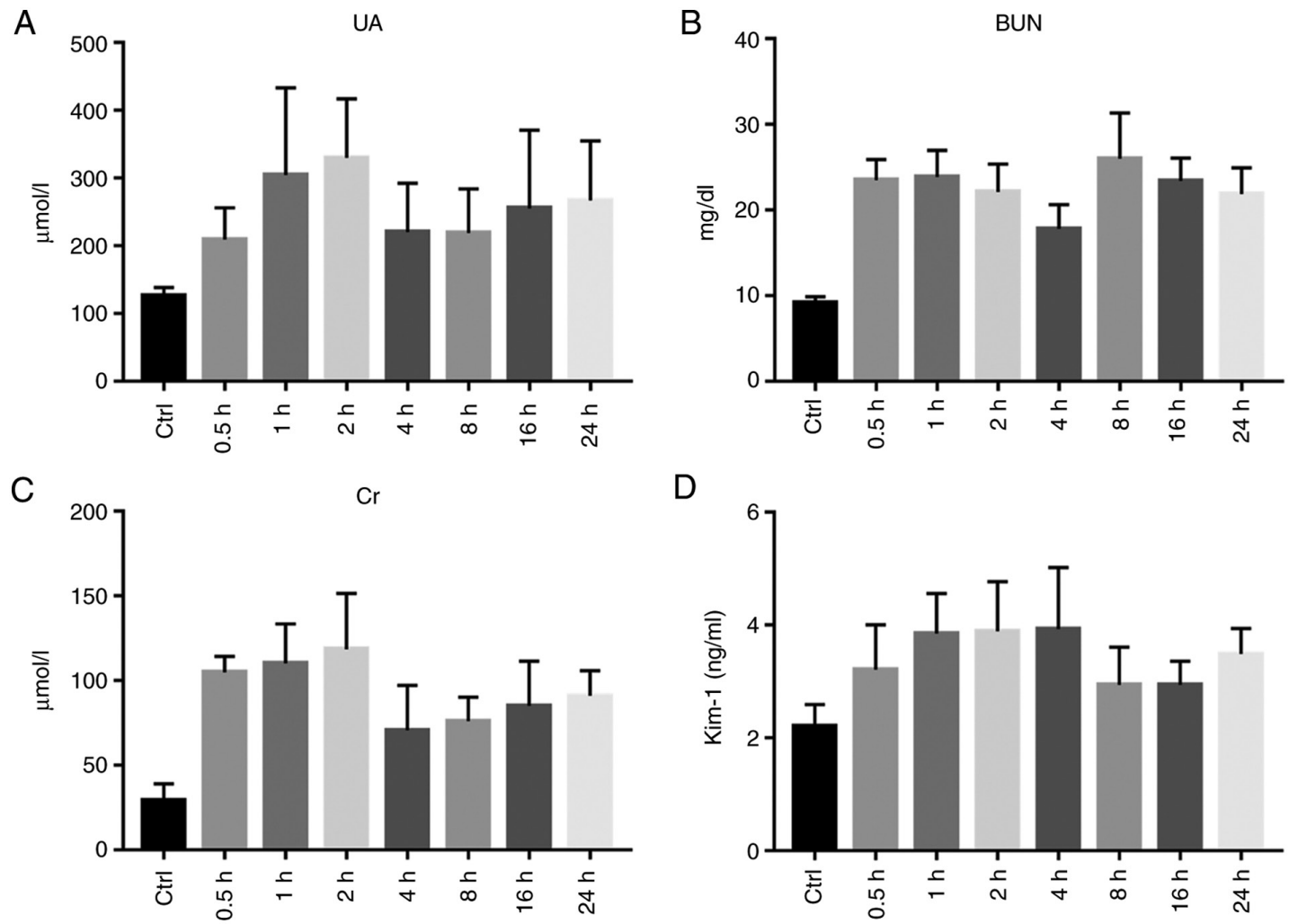

Figure 7. Renal function. (A) Statistical chart of serum uric acid (UA) level in the Control (Ctrl) and Experimental group (at $0.5,1,2,4,8,16$ and $24 \mathrm{~h}$ after diquat exposure). (B) Statistical chart of serum urea nitrogen (BUN) level in the Control (Ctrl) and Experimental group (at $0.5,1,2,4,8,16$ and $24 \mathrm{~h}$ after diquat exposure). (C) Serum creatinine (Cr) level of rats in the Control (Ctrl) and Experimental group (at 0.5, 1, 2, 4, 8, 16 and $24 \mathrm{~h}$ after diquat exposure). (D) Statistical chart of serum kidney injury molecule-1 (KIM-1) content in the Control (Ctrl) and Experimental group (at 0.5, 1, 2, 4, 8, 16 and $24 \mathrm{~h}$ after diquat exposure).

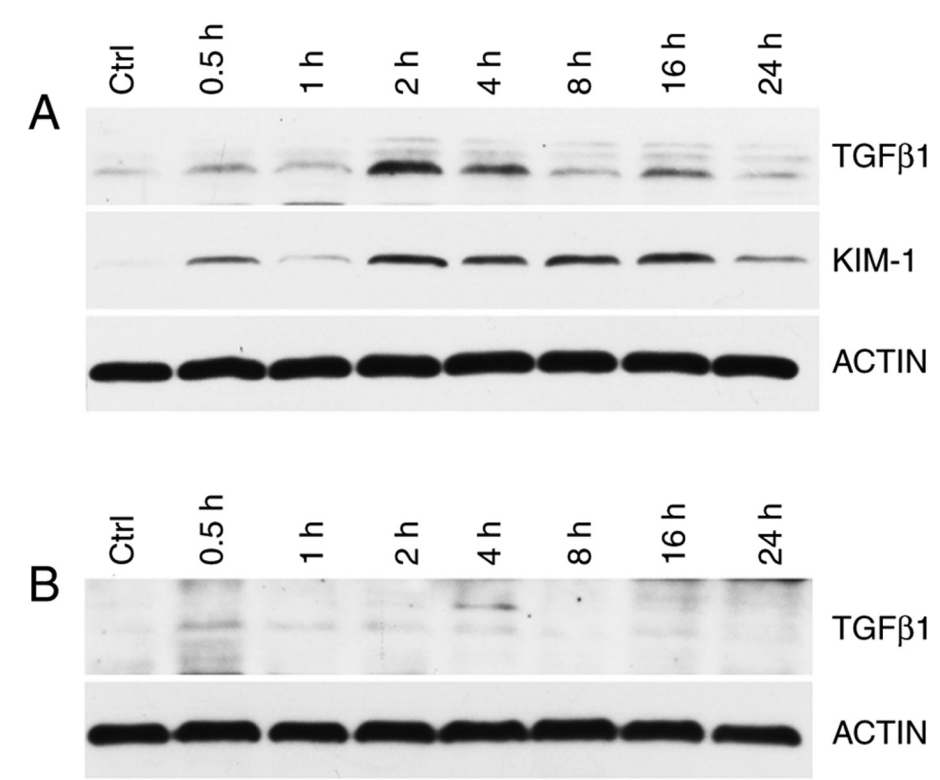

Figure 8. (A) Western blot grayscale images of KIM-1 and TGF- $\beta 1$ in rat kidney in the Control (Ctrl) and Experimental group (at 0.5, 1,2,4, 8, 16 and $24 \mathrm{~h}$ after diquat exposure). (B) Western blot grayscale images of TGF- $\beta 1$ in rat lung tissue in the Control and Experimental group. KIM-1, kidney injury molecule-1; TGF- $\beta 1$, tumor growth factor- $\beta 1$.

Kidney injury molecule-1 (KIM-1) is a novel potential urinary biomarker for the early detection of acute kidney injury (AKI) within $24 \mathrm{~h}$ following kidney insult $(13,14)$. The serum levels of KIM-1 were tested in the rats following exposure; they began to increase at $0.5 \mathrm{~h}$ and significantly increased at $4 \mathrm{~h}$. This indicated that KIM-1 may serve as an early biomarker of kidney damage caused by early diquat poisoning. Although none of the reported biomarkers are entirely specific for 
AKI (15), biomarkers such as KIM-1 may be positively used for the risk stratification of diquat exposure-induced AKI.

Tumor growth factor (TGF)- $\beta 1$ as a multifunctional growth factor, is closely associated with the deposition of the extracellular matrix and is an important regulator of glomerulosclerosis and renal tubule and interstitial fibrosis. Additionally, evidence indicates the causal relationship between the increase in TGF- $\beta 1$ levels and tissue fibrosis $(12,16,17)$. The present study indicated that TGF- $\beta 1$ was strongly expressed in renal tissues of the diquat-exposed mice, but was scarcely expressed in lung tissues. In addition, its expression was mainly concentrated at 2 and $4 \mathrm{~h}$ when compared to other time period in the kidneys, and the levels of expression may have been positively correlated with the degree of renal interstitial fibrosis. TGF- $\beta 1$ in the kidney tissues was therefore significantly higher. TGF- $\beta 1$ may be linked to the mechanism of AKI caused by diquat poisoning.

Histological analysis of the H\&E-stained kidney sections indicated that the renal tubules were the primary targets of injury, with renal tubular epithelial cells showing hydropic degeneration, and a small number of casts observed in the lumen. KIM-1 is characteristically expressed in the epithelial cell parietal membranes of the renal proximal convoluted tubules. In the present study, KIM-1 began to increase $0.5 \mathrm{~h}$ after exposure, which supported the interpretation that renal injury was likely initiated principally in the proximal tubules. Electron microscopy analysis of renal tissues showed that the renal tubular epithelial cells in each group had relatively complete cell membranes, basically complete organelle structures and an abundance of organelles in the cytoplasm. However, after $2 \mathrm{~h}$, there were more vacuoles (suspected to be formed by the swelling of mitochondria or formed after autophagic digestion), the local nuclear membrane and mitochondrial cristae were indistinct, and autophagy was increased. Heterochromatin levels increased after $4 \mathrm{~h}$. The mitochondria swelled and the cytoplasm was vacuolated. After $24 \mathrm{~h}$, the number of vacuoles increased compared to that at $16 \mathrm{~h}$. Some of the mitochondrial bilayer membrane structures disappeared, and the number of lysosomes increased. Collectively, these results strongly suggested that diquat exposure induces mitochondrial dysfunction. Diquat-induced cell death in the kidney of rats leads to kidney dysfunction, and the degree of damage may gradually increase over time.

The damage caused to lungs by diquat within $24 \mathrm{~h}$ was determined by detecting the hydroxyproline (HYP) levels in the lung tissue. As one of the main components of collagen in the body, HYP accounts for $13.4 \%$ of collagen amino acids, a very small amount of elastin (18). The HYP content in the lung tissue is a direct indicator of the degree of pulmonary fibrosis. When pulmonary fibrosis occurs, the amount of collagen fibers in the lung tissue increases. In rats exposed to diquat, the lack of increase in HYP content in the lung tissues showed that there was no marked difference in lung tissue injury and lung function between the exposure and control groups. Diquat caused relatively less injury to the lungs and did not lead to any obvious formation of fibrotic scars in the lung tissues at the early stages after exposure. However, whether the degree of pulmonary fibrosis worsens with time remains to be confirmed by further experimental studies.
In conclusion, in the present study, an animal model of acute diquat exposure was established, and the changes in diquat in the plasma and organ tissues were dynamically observed. The pathological damage to the kidney and lung tissues caused by diquat was studied. The results demonstrated that the diquat content in the organs and tissues was related to organ injury; although the mechanisms of kidney injury are not completely understood, the release of inflammatory factors is believed to play an important role, and the related acute exposure-related mechanism requires further exploration. By determining the main organs damaged by diquat poisoning, the patterns of plasma and tissue concentration of diquat and organ damage were preliminarily explored in order to provide an experimental basis for clinical preventive interventions and effective therapeutic options of diquat poisoning.

As a limitation of the present study, there was not enough data that could accurately determine the clinical prognosis based on the measured plasma diquat concentration. The pharmacokinetics of diquat in the human body can only be inferred based on the results of animal experiments to date. It is preliminarily estimated that the best time is the early clinical treatment of patients. The relationship between the blood concentration of diquat and the prognosis of patients requires us to conduct further research through clinical epidemiological observations.

\section{Acknowledgements}

Not applicable.

\section{Funding}

No funding was received.

\section{Availability of data and materials}

The datasets used and/or analyzed during the current study are available from the corresponding author on reasonable request.

\section{Authors' contributions}

YW, BK and XJ conceived and designed the study. YW, SC, WW, TJ and XJ performed the experiments. YW, SC and BK wrote the paper. SC, BK and $\mathrm{XJ}$ reviewed and edited the manuscript. BK and XJ critically revised important intellectual content. YW and XJ confirm the authenticity of all the raw data All authors read and approved the manuscript and agree to be accountable for all aspects of the research in ensuring that the accuracy or integrity of any part of the work are appropriately investigated and resolved.

\section{Ethics approval and consent to participate}

All experiments were conducted following the guidelines of the Chinese Laboratory Animal Welfare Ethical Review. Ethical approval was provided by the Ethics Committee of Preventive Medicine of Shandong University (no. 20180225) for this study. 


\section{Patient consent for publication}

Not applicable.

\section{Competing interests}

The authors declare that they have no competing interests.

\section{References}

1. Ameno K, Fuke C, Shirakawa Y, Ogura S, Ameno S, Kiriu T, Kinoshita $\mathrm{H}$ and Ijiri I: Different distribution of paraquat and diquat in human poisoning cases after ingestion of a combined herbicide. Arch Toxicol 68: 134-137, 1994.

2. Van Vleet TR and Schnellmann RG: Toxic nephropathy: Environmental chemicals. Semin Nephrol 23: 500-508, 2000.

3. Jones GM and Vale JA: Mechanisms of toxicity, clinical features, and management of diquat poisoning: A review. J Toxicol Clin Toxicol 38: 123-128, 2000

4. Fortenberry GZ, Beckman J, Schwartz A, Prado JB, Graham LS, Higgins S,Lackovic M, Mulay P, Bojes H, Waltz J, et al: Magnitude and characteristics of acute paraquat- and diquat-related illnesses in the US: 1998-2013. Environ Res 146: 191-199, 2016.

5. Magalhães N, Carvalho F and Dinis-Oliveira RJ: Human and experimental toxicology of diquat poisoning: Toxicokinetics, mechanisms of toxicity, clinical features, and treatment. Hum Exp Toxicol 37: 1131-1160, 2018.

6. Dinis-Oliveira RJ, Duarte JA, Sánchez-Navarro A, Remião F, Bastos ML and Carvalho F: Paraquat poisonings: Mechanisms of lung toxicity, clinical features, and treatment. Crit Rev Toxicol 38: 13-71, 2008.

7. Fussell KC, Udasin RG, Gray JP, Mishin V, Smith PJ, Heck DE and Laskin JD: Redox cycling and increased oxygen utilization contribute to diquat-induced oxidative stress and cytotoxicity in Chinese hamster ovary cells overexpressing NADPH-cytochrome P450 reductase. Free Radic Biol Med 50: 874-882, 2011.
8. Vanholder R, Colardyn F, De Reuck J, Praet M, Lameire N and Ringoir S: Diquat intoxication: Report of two cases and review of the literature. Am J Med Jun 70: 1267-1271, 1981.

9. Clark DG and Hurst EW: The toxicity of diquat. Br J Ind Med 27: $51-55,1970$.

10. Rose MS, Crabtree HC, Fletcher K and Wyatt I: Biochemical effects of diquat and paraquat. Disturbance of the control of corticosteroid synthesis in rat adrenal and subsequent effects on the control of liver glycogen utilization. Biochem J 138: 437-443, 1974

11. Lock EA: The effect of paraquat and diquat on renal function in the rat. Toxicol Appl Pharmacol 48: 327-336, 1979.

12. Zhang J, Zhao Y, Bai Y, Lv G, Wu J and Chen Y: The significance of serum uric acid level in humans with acute paraquat poisoning. Sci Rep 5: 9168, 2015.

13. Djukic M, Jovanovic MD, Ninkovic M, Stevanovic I, Curcic M, Topic A, Vujanovic D and Djurdjevic D: Intrastriatal pre-treatment with L-NAME protects rats from diquat neurotoxcity. Ann Agric Environ Med 19: 666-672, 2012.

14. Rogers LK, Bates CM, Welty SE and Smith CV: Diquat induces renal proximal tubule injury in glutathione reductase-deficient mice. Toxicol Appl Pharmacol 217: 289-298, 2006.

15. Kashani K, Cheungpasitporn W and Ronco C: Biomarkers of acute kidney injury: The pathway from discovery to clinical adoption. Clin Chem Lab Med 55: 1074-1089, 2017.

16. Calvier L, Chouvarine P, Legchenko E, Hoffmann N, Geldner J, Borchert P, Jonigk D, Mozes MM and Hansmann G: PPAR $\gamma$ Links BMP2 and TGF- $\beta 1$ pathways in vascular smooth muscle cells, regulating cell proliferation and glucose metabolism. Cell Metab 25: 1118-1134.e7, 2017.

17. Kim KK, Sheppard D and Chapman HA: TGF- $\beta 1$ signaling and tissue fibrosis. Cold Spring Harb Perspect Biol 10: a022293, 2018.

18. Wu Z, Hou Y, Dai Z, Hu CA and Wu G: Metabolism, nutrition, and redox signaling of hydroxyproline. Antioxid Redox Signal 30: 674-682, 2019.

(i) (8) $\Theta$ This work is licensed under a Creative Commons Attribution-NonCommercial-NoDerivatives 4.0 International (CC BY-NC-ND 4.0) License. 